Angeles Costa, M. de los p36

Arranz, C. p36

Bacic, D. p76

Balaszczuk, A.M. p36

Beerenhout, C.M. p43

Biber, J. p76

Braunewell, K.-H. p76

Connors, B.A. p67

Cremades, A. p57

Dammers, R. p43

Darmon, D. p1

Eisner, G.M. p19

Evan, A.P. p67

Felder, R.A. p19

Fellet, A. p36

Fineberg, N.S. p67

García, J. p36
Gilbert, R.E. p83

Gisler, S. p76

Gladziwa, U. p43

Goldfarb, M. p1

Greven, J. p49

Gurina, N.M. p10

Heyman, S.N. p1

Hoeks, A.P.G. p43

Ivanov, A.I. p10

Jeyarajah, D.R. p28

Jose, P.A. p19

Kelly, D.J. p83

Kielar, M.L. p28

Konings, C.J.A.M. p43

Kooman, J.P. p43

Kuo, R.L. p67

Leunissen, K.M.L. p43
Lifshitz, D.A. p67

Lindup, W.E. p10

Lingeman, J.E. p67

Loffing, J. p76

Lu, C.Y. p28

Madjdpour, C. p76

Maslenny, V.N. p10

Mifsud, S. p83

Murer, H. p76

Nieman, F.H.M. p43

Nikolaev, V.G. p10

Paterson, R.F. p67

Peñafiel, R. p57

Pollock, C.A. p83

Pribanic, S. p76

Qi, W. p83

Rensma, P.L. p43
Rosen, S. p1

Sande, F.M. van der p43

Sarnatskaya, V.V. p10

Schlattjan, J.H. p49

Shalhav, A.L. p67

Shina, A. p1

Simon, J.R. p67

Tjia, J. p10

Tomat, A. p36

Vicente-Ortega, V. p57

Wilkinson-Berka, J.L. p83

Willis, L.R. p67

Winter, C. p49

Yushko, L.A. p10

Zhang, Y. p28

Zhou, X.J. p28

\title{
Subject Index Vol. 95, 2003
}

Activated carbon p10

Acute renal failure p28

Amiloride p57

3-Aminobenzamide $\mathrm{p} 1$

Apical sodium-dependent bile salt transporter, ASBT p49

Arterial distensibility p43

- stiffness p43

Atrophy p83

Biliary obstruction p28

Blood pressure regulation p19

Cholestasis p49

Clearances, ${ }^{3} \mathrm{H}$-taurocholate and ${ }^{3} \mathrm{H}$-cholate $\mathrm{p} 49$

Denervation p67

Dopamine receptor p19
Fibrosis p83

G protein-coupled receptor kinase $\mathrm{p} 19$

Glomerular filtration rate $\mathrm{p} 49$

Human albumin p10

Hyperkalemia p57

Hypertension p36

Interacting proteins $\mathrm{p} 76$

Interleukin-1 receptor antagonist $\mathrm{p} 28$

Interleukin-10 p28

Kidney failure, acute $\mathrm{p} 1$

Lithotripsy p67

Medulla p1

Medullary thick ascending limb $\mathrm{p} 1$
Melting curve p10

Mouse kidney p57

$\mathbf{N a} / \mathrm{Pi}$ cotransport p76

Nephrectomy p36

Nephropathy $\mathrm{p} 83$

Neuronal calcium sensors p76

Nitric oxide p36

- - synthase p36

Original renal disease p43

Poly(ADP-ribose) polymerase p1

Potassium p57

Predictors p43

Prostaglandins $\mathrm{p} 1$

Proximal tubular cells p49

Radiologic contrast media $\mathrm{p} 1$
Rat p1

Renal failure $\mathrm{p} 43$

- hemodynamics p67

- nerves p67

- sexual dimorphism p57

- toxicity p57

Renorenal reflex p67

Shockwaves p67

Taurocholate p49

Testosterone p57

Tranilast p83

Transforming growth factor- $\beta$ p83

Tubulointerstitium p83

Uraemia p10

Yeast two-hybrid p76

\section{KARGER}

(C) 2003 S. Karger AG, Basel

Fax +4161306 1234

E-Mail karger@karger.ch

www.karger.com
Accessible online at:

www.karger.com/nep 